\title{
Incidencia del laboratorio de Ciencias Naturales en los estudiantes de URACCAN
}

\author{
Incidence of the Natural Sciences Laboratory in URACCAN students
}

\author{
Carlos Álvarez Amador ${ }^{1}$ \\ Elder Alfonso González Brizuela² \\ Arsenio López Borge ${ }^{3}$
}

\section{Resumen}

Actualmente se discute el quehacer de los Laboratorios de Ciencias Naturales y es difícil entender cuáles son sus aportes, pues su labor depende de diversos factores. El trabajo de estos en el recinto URACCAN Nueva Guinea no es ajeno a tal afirmación. Con esta investigación se visualiza la incidencia de dichos espacios en la formación del estudiantado y por ende el logro de la misión de la universidad.

La investigación fue desde una perspectiva de enfoque mixto, predominantemente cuantitativo, de tipo descriptiva con una población de 268 estudiantes y 16 docentes de 6 carreras. La muestra para estudiantes fue de 71, calculada con el STATTM. En el caso de los docentes se realizó un muestreo al 100\% (censo). La población de estudiantes se estratificó por carreras y por género. Se utilizaron la encuesta, entrevista y revisión documental. Se definieron como grandes variables la pertinencia, calidad, impacto y sostenibilidad de las prácticas del Laboratorio de Ciencias Naturales.

Se constató incidencia significativa del Laboratorio de Ciencias Naturales en el proceso educativo del estudiantado y aportes a la docencia, con un promedio general de $82 \%$, resaltando la vinculación de la teoría con la práctica, la utilidad de los conocimientos adquiridos para el desempeño académico y profesional para fortalecer su capacidad de análisis, desarrollar habilidades y destrezas, entre otras. Se lograron identificar las potencialidades del laboratorio y algunas limitantes que son parte de la realidad de este.

Los procesos y actividades de formación desarrolladas en el laboratorio han aportado de manera efectiva, eficiente y con calidad, favoreciendo el aprendizaje y contribuyendo al fortalecimiento de las capacidades docentes. Pese a ello, se recomienda la readecuación y ampliación de espacios y dotación de recursos (equipos, materiales y reactivos).

Palabras clave: Laboratorio; ciencias naturales; aporte del laboratorio; enseñanza-aprendizaje; incidencia.

\section{Abstract}

Currently, the work of the Natural Science Laboratories is discussed and it is difficult to understand what their contributions are, since their work depends on various factors. The work of these at URACCAN Nueva Guinea campus is no stranger to such a statement. With this research, the incidence of these spaces in the student formation and, therefore, the achievement of the university's mission is visualized.

\footnotetext{
1 Ingeniero agroforestal, Máster en Docencia Universitaria, docente de la URACCAN, recinto Nueva Guinea, email: caxy123@yahoo.com Orcid: http://orcid. org/0000-0001-8588-4471

2 Licenciado en química, Máster en Docencia Universitaria, docente de la URACCAN, recinto Nueva Guinea, email: egonzalezbrizuela@yahoo.es Orcid: http:// orcid.org/0000-0002-2368-9790

3 Ingeniero agro ecólogo, Master en Docencia Universitaria, docente de la URACCAN, recinto Nueva Guinea, email: albsiuna@gamil.com
} 
The research was from a perspective of mixed, predominantly quantitative, descriptive approach with a population of 268 students and 16 teachers of 6 careers. The sample for students was 71, calculated with the STATTM. In the case of teachers, a 100\% sampling (census) was carried out. The student population was stratified by careers and gender. The survey, interview and documentary review were used. The relevance, quality, impact and sustainability of the practices of the Natural Sciences Laboratory were defined as outsized variables.

There was a significant incidence of the Natural Sciences Laboratory in the student's educational process and contributions to teaching, with a general average of $82 \%$, highlighting the link between theory and practice, the usefulness of acquired knowledge for academic and professional performance to strengthen their ability to analyze, develop skills and abilities, among others. They were able to identify the potential of the laboratory and some limitations that are part of the reality of this.

The training processes and activities developed in the laboratory have contributed effectively, efficiently and with quality, favoring learning and contributing to the strengthening of teaching capacities. Despite this, the readjustment and expansion of spaces and provision of resources (equipment, materials and reagents) is recommended.

Keywords: Laboratory; Natural Sciences; laboratory input; teaching - learning; incidence.

\section{Introducción}

La educación superior como un proceso de formación en el que se prepara el individuo para satisfacer unos requerimientos en un mundo cada vez más exigente, provista de diversos medios y estrategias para lograr una formación acorde a tales exigencias. Ello ha supuesto que en el diseño de los currículos de las carreras profesionales se ponga especial énfasis al logro de las competencias y que estas se traduzcan en habilidades y destrezas de los profesionales formados con tales diseños curriculares.

La revolución científica y tecnológica que la humanidad ha generado es uno de los factores que influyen en el desarrollo de la educación superior, ello ha llevado a que las universidades se preocupen cada vez más por modernizar sus medios de enseñanza, tales como laboratorios de Ciencias Naturales, espacios en los que los estudiantes aprenden haciendo, estos se traducen en verdaderas oportunidades de logro para la calidad que lógicamente toda universidad persigue.

Actualmente se discute el trabajo de los laboratorios y es difícil entender cuáles son sus aportes. Pues la labor de estos depende de factores como: el enfoque de enseñanza, el tipo de actividad, el tipo de instrumento de evaluación, el nivel educativo al que se dirige la instrucción, el currículo a desarrollar, la correspondencia entre objetivos que se pretenden lograr y cómo pretende lograrse. Además, hay que considerar que una visión reduccionista del trabajo práctico del laboratorio entra en contradicción con una visión holista del mismo, por ello los objetivos del laboratorio están sujetos en primera instancia a la visión que tiene el docente, sin obviar la propia visión de los estudiantes, que muchas veces no es la misma, como lo han podido demostrar investigaciones en el área (Flores et al., 2009).

La universidad URACCAN no es ajena a la premisa de garantizar una educación superior de calidad, esto se observa en su misión, la cual es: formar hombres y mujeres con conocimientos, saberes, capacidades, valores, principios, actitudes humanistas, sentido de emprendimiento e innovación, en equilibrio y armonía con la Madre Tierra para el fortalecimiento de las Autonomías de los Pueblos (URACCAN, 2014).

Con la realización de esta investigación se pretende visibilizar el trabajo del Laboratorio de Ciencias Naturales a la formación del estudiantado y por ende al logro de la misión de la universidad. 


\section{Revisión de literatura}

\section{Definición de laboratorio}

En términos generales, Lugo (2006) define un laboratorio como:

Un lugar equipado con diversos instrumentos de medición, entre otros, donde se realizan experimentos o investigaciones diversas, según la rama de la ciencia a la que se enfoque. Dichos espacios se utilizan tanto en el ámbito académico como en la industria y responden a múltiples propósitos, de acuerdo con su uso y resultados finales, sea para la enseñanza, para la investigación o para la certificación de la industria. (p. 20)

\section{Importancia de los laboratorios}

La importancia de los laboratorios tanto en la enseñanza de las ciencias como en la investigación y en la industria es, sin duda alguna, indiscutible.

Lugo (2006) expresa que:

No se puede negar que el trabajo práctico en laboratorio proporciona la experimentación y el descubrimiento y evita el concepto de "resultado correcto" que se tiene cuando se aprenden de manera teórica, es decir, sólo con los datos procedentes de los libros. (p. 20)

Sin embargo, Urrea et al. (2013) sostienen que "el uso de laboratorios requiere de tiempo adicional al de una clase convencional, por ejemplo, para descubrir y aprender de los propios errores". La revolución científico técnica, precisa poner al alcance de los estudiantes en general los conocimientos científicos, y en ello juega un papel fundamental el experimento docente, como parte inseparable de su formación cultural, para su futura inserción en la vida (Colado, s.f.).

En la enseñanza general al estudiante se le plantean tareas nuevas y cada vez más complejas, cuya solución exige que sea capaz de orientarse adecuadamente en relación con los objetos y fenómenos, esto es, que pueda distinguir sus cualidades y establecer relaciones entre ellos. Es decir, que toda tarea práctica implica una correcta percepción de los objetos, sus cualidades y sus relaciones y esto se logra con el uso de los laboratorios y sus recursos a la disposición del estudiantado (Colado, s.f.).

Los aportes que la experimentación y la actividad práctica hacen en la formación de conocimientos, habilidades, hábitos, actitudes y valores desde la primaria hasta la culminación de sus estudios, constituyen una fuente importante en la formación del individuo, que dejará una huella imperecedera en él y que, en gran medida, será decisiva en su ulterior desarrollo, así como en su proyección ante la vida y su concepción del mundo. Para ello debemos realizar una actividad experimental, que en realidad le permita al estudiante una verdadera reflexión de los objetos y fenómenos estudiados y su aplicación a su contexto cotidiano (Colado, s.f.).

\section{Aporte de los laboratorios en diversas áreas de la ciencia}

El Gobierno de Córdoba, Ministerio de Educación, Secretaría de Educación, Subsecretaría de Estado de Promoción de Igualdad y Calidad Educativa (2015) realizaron un estudio documental en Argentina y concluyeron que las actividades experimentales constituyen uno de los aspectos claves en el proceso de enseñanza y aprendizaje de las Ciencias Naturales dado que contribuyen a la comprensión de los hechos y fenómenos del ambiente y colaboran con que se aprenda a disfrutar de las ciencias.

Alonzo y Rodríguez (1990), afirman que muchos autores concuerdan en que los laboratorios, sobre todo académicos, aportan de la siguiente manera: 
1. Despertar el interés de los estudiantes hacia las ciencias

2. Desarrollar habilidad y confianza en el manejo de equipo

3. Ilustrar y reforzar la teoría

4. Aplicar el método científico en la resolución de problemas.

Por otro lado, Lugo (2006) afirma que, en Estados Unidos, diversas universidades y escuelas de graduados cuentan con laboratorios equipados con aparatos de investigación desde los moderados hasta los más avanzados en cuanto a tecnología, para responder a las necesidades de ese país en términos de investigación y entrenamiento de futuros científicos y académicos universitarios.

En otro aspecto, el aporte específico en el campo de las ingenierías es mayormente tangible. Lugo (2006), afirma que:

En los laboratorios de ingeniería se muestran los fenómenos físicos básicos, se brindan a los estudiantes experiencias directas donde pueden lograr la experiencia para ejercer la ingeniería. Ejemplo de ello son los laboratorios de Diseño de Concreto Reforzado y de Materiales de Construcción donde a los estudiantes de las ingenierías Civil, Arquitectónica y del Medio Ambiente se les proporciona la práctica para la mejor comprensión de los diferentes conceptos. (p. 22)

En síntesis, Scoles y Patticini como se citó en Urrea et al. (2013), concluyen que la aplicación de estas alternativas de aprendizaje viene a ser una opción frente al desarrollo de la enseñanza tradicional, en la que prevalece la clase magistral. La enseñanza tradicional no resulta completamente eficaz para un aprendizaje significativo, recomendando el empleo de métodos menos pasivos para el estudiante, afirmando que los mismos perciben el laboratorio como un lugar donde están activos.

Por su parte Hodson (1994), remarca que innovar en las prácticas de laboratorio teniendo en cuenta lo que el alumno ya sabe juega un papel fundamental en lo que este aprende. Lo anterior sustenta la afirmación de que las prácticas de laboratorio juegan un papel primordial en la familiarización de los estudiantes con la metodología científica (Salcedo et al., 2005).

\section{Los propósitos del trabajo de laboratorio}

Mucho se ha dicho acerca de los objetivos del trabajo de laboratorio. Alonzo y Rodríguez (1990), sostienen que hay cuatro razones fundamentales por las cuales es necesario emplear el método del trabajo de laboratorio:

1. La mayoría de los estudiantes, independientemente de su edad cronológica, fracasan cuando se trata de comprender conceptos científicos, de tipos complejos y abstractos, sin la ayuda de objetos reales y la oportunidad para manipularlos.

2. El trabajo de laboratorio permite que los estudiantes tomen parte en el método de la ciencia y, sobre todo, que tengan la vivencia del espíritu científico.

3. La experiencia práctica propicia el desarrollo de habilidades que pueden ser generalizadas a una amplia variedad de asignaturas.

No obstante, Barberá y Valdés (1996) afirman que:

La definición de los objetivos del trabajo de laboratorio ha sido un punto de discusión difícil de esclarecer y es actualmente un área de investigación activa. La labor depende de múltiples factores, entre los que se pudieran citar: el enfoque de enseñanza, el tipo de actividad, el tipo de instrumento de evaluación, el nivel educativo al que se dirige la instrucción, el currículo a desarrollar, la correspondencia entre objetivos que se pretenden lograr y cómo pretende lograrse. (p. 79) 
Las prácticas de laboratorio brindan a los estudiantes la posibilidad de entender cómo se construye el conocimiento dentro de una comunidad científica, cómo trabajan los científicos, cómo llegan a acuerdos y cómo reconocen desacuerdos, qué valores mueven la ciencia, cómo se relaciona la ciencia con la sociedad y la cultura (López \& Tamayo, 2012).

El objetivo principal de la práctica de laboratorio es facilitar que los alumnos lleven a cabo sus propias investigaciones, se contribuye a desarrollar la comprensión sobre la naturaleza de la ciencia y su reflexión sobre el propio aprendizaje personal (Cardona, 2013).

Kirschner (1992) los condensa en tres motivos, los cuales él mismo cuestiona:

1. La práctica sirve a la teoría científica, por lo que se centra en actividades verificativas, experimentos a prueba de errores y manipulación de aparatos, lo cual no contribuye a comprender la naturaleza sintáctica de las disciplinas científicas, es decir, los hábitos y destrezas de quienes la practican.

2. Se le ha atribuido al descubrimiento una asociación con el aprendizaje significativo, lo cual no tiene fundamento filosófico ni pedagógico, de acuerdo con Hodson, 1994.

3. El trabajo empírico con el mundo de los fenómenos brinda insight y comprensión; esto se cuestiona por el hecho de que la observación requiere de una estructura conceptual del observador; en otras palabras, el significado de los conceptos no está en la experiencia sino viceversa, el significado de la experiencia está en los conceptos que tiene el individuo. Esto permite comprender, en cierto modo la explicación que los estudiantes dan a fenómenos observados en su vida cotidiana no coincide con las explicaciones científicas construidas sobre la base de conceptos y teorías abstractas.

Hasta finales de los años cincuenta del pasado siglo, la enseñanza del laboratorio se centró principalmente en actividades verificativas discutidas en las clases de teoría, planteadas en los libros de texto o sugeridas en manuales de laboratorio. Según Hofstein y Lunetta (2004) esta situación se trató de cambiar con el nuevo curriculum de los años sesenta, dándosele a la enseñanza del laboratorio la función importante de desarrollar habilidades de alto nivel cognitivo, mediante actividades centradas en los procesos de la ciencia a través del método indagatorio.

Sin embargo, Barberá y Valdés (1996) señalan que, investigaciones de los años sesenta revelaron que los estudiantes, profesores, investigadores y diseñadores curriculares, en los diversos niveles educativos, no coincidían con relación a los objetivos del laboratorio. Asimismo, algunos estudios indican que los objetivos del laboratorio cambian de acuerdo con el nivel educativo, habiendo mayor unanimidad al respecto en los niveles más bajos que en los superiores de la enseñanza secundaria, por lo que se pudiera esperar que la discrepancia sea mayor a nivel universitario (Hodson, 1994).

En este contexto, vale la pena señalar que Woolnough y Allsop como se citó en Barberá y Valdés (1996) plantearon, a mediados de los ochenta, tres objetivos que se orientan a la enseñanza de la estructura sintáctica de la ciencia. Estos objetivos son: (a) desarrollar técnicas y destrezas prácticas a través de ejercicios; (b) tomar conciencia de fenómenos naturales a través de experiencias; y (c) resolver problemas científicos en actividades abiertas a través de investigaciones. Esta clasificación permite planificar actividades específicas de laboratorio de acuerdo con los objetivos que se pretendan lograr, considerando el nivel de complejidad cognitiva requerida y/o deseada.

Aunque el planteamiento de Woolnough y Allsop responde a objetivos propios del laboratorio, Barberá y Valdés (1996) propusieron en los noventa cuatro objetivos que consideraron característicos del trabajo práctico porque pueden lograrse sólo a través del mismo. Estos objetivos se seleccionaron de clasificaciones realizadas por otros autores: (a) Proporcionar experiencia directa sobre fenómenos, (b) permitir contrastar la abstracción científica ya establecida con la realidad que pretende describir, (c) desarrollar competencias técnicas y (d) Desarrollar el razonamiento práctico. 
Por ende, los aspectos conceptuales, procedimentales y epistemológicos involucrados en el trabajo de laboratorio constituyen la base de las investigaciones que se pueden continuar desarrollando sobre el rol del laboratorio en la enseñanza de las ciencias.

Según Kirschner (1992), el trabajo práctico se debe utilizar para enseñar y aprender la estructura sintáctica de una disciplina, más que la estructura sustantiva. Plantea tres razones o motivos válidos para ello: (a) Desarrollar destrezas específicas a través de ejercicios; (b) Aprender el "enfoque académico" a través de los trabajos prácticos como investigaciones, de modo que el estudiante se involucre en la resolución de problemas como lo hace un científico; y (c) Tener experiencias con fenómenos. El trabajo práctico como una situación de investigación permite desarrollar destrezas en la resolución de problemas, y esto implica: (a) Reconocer la existencia de un problema en una situación dada; (b) Definir el problema; (c) Buscar soluciones alternativas; (d) Evaluar las soluciones alternativas; (e) Escoger la mejor estrategia de solución; y (f) Evaluar la solución para ver si hay nuevos problemas volviendo al principio.

\section{La efectividad del trabajo de laboratorio}

Según Hodson (1994), una posible explicación de las pocas ventajas que se le han atribuido a los trabajos prácticos radica en seis razones: (a) No se discriminan los tipos de "trabajos prácticos", porque se colocan todos en una misma categoría; (b) El trabajo práctico generalmente es pobremente diseñado y ejecutado; (c) La retórica de los docentes no se corresponde con su práctica; (d) Los estudiantes no atienden las instrucciones de los docentes en la forma que se espera; (e) El trabajo práctico no siempre resulta de la manera esperada, dando así resultados erróneos o ningún resultado; y (f) La evaluación se hace sobre aspectos menos importantes de la actividad.

Además, algunas investigaciones realizadas con relación a la efectividad de los trabajos de laboratorio han sido muy cuestionadas por ser metodológicamente confusas, generando pocos resultados concluyentes (Hodson, 1994; Barberá y Valdés, 1996). Esto podría atribuírsele, en gran parte, al limitado enfoque cuantitativo con el que se desarrollaron y a la falta de consenso sobre los objetivos de los trabajos de laboratorio.

Hasta el momento se puede decir que la mayoría de las investigaciones apoyan las ventajas de los estilos o enfoques alternativos al tradicional (excepto el método por descubrimiento), aunque es necesario indagar sobre aspectos más específicos que permitan dilucidar la relación entre los diferentes enfoques y resultados particulares de aprendizaje.

\section{Contexto institucional de los laboratorios de Ciencias Naturales}

\section{Marco filosófico de la URACCAN}

La universidad URACCAN en su perfil institucional, es un proyecto institucional de universidad propia, articulado de conformidad a las aspiraciones históricas de los pueblos y comunidades indígenas, afrodescendientes y mestizos del Caribe por gozar de un modelo de desarrollo construido desde las bases socioculturales (URACCAN, 2008). Por otro lado, URACCAN (2004) indica que su filosofía institucional está orientada a revitalizar, promover y fortalecer el ejercicio de derechos colectivos, identidades, cosmovisiones, espiritualidades, formas de organización, culturas, sistemas de conocimientos y prácticas. Esto se da al mismo tiempo de evitar la fuga de talentos humanos, fortalecer la Autonomía Regional Multiétnica y el Sub-sistema Educativo Autonómico Regional.

Su misión y visión contextualizada a los nuevos retos del milenio (URACCAN, 2014) se centran en: Misión: Formar hombres y mujeres con conocimientos, saberes, capacidades, valores, principios, actitudes humanistas, sentido de emprendimiento e innovación, en equilibrio y armonía con la Madre Tierra para el fortalecimiento de las Autonomías de los Pueblos. Visión: Ser líder en el modelo de Universidad Comunitaria Intercultural reconocida a nivel regional, nacional e internacional por su calidad y pertinen- 
cia, que acompaña procesos de gestión e incidencia, para la construcción de ciudadanías interculturales de género, que conlleven al Buen Vivir y la Autonomía de los Pueblos indígenas, afrodescendientes, mestizos y otras comunidades étnicas.

\section{El modelo pedagógico para la construcción de conocimientos}

La URACCAN se fundamenta en acciones que llevan a recrear, crear, compartir, diseminar y al intercambio de conocimientos, saberes y prácticas desde dos vías: la interacción entre el conocimiento local ancestral y el conocimiento occidental en lo económico, productivo, social, cultural y ambiental que conlleve a la producción de nuevas teorías. La Universidad orienta sus esfuerzos hacia la construcción y reconstrucción de conocimientos, apoyados en las prácticas y saberes endógenos. Mediante la investigación, vincula las prácticas de las aulas con la realidad objetiva del entorno a través de un proceso participativo, autogestionario y dinámico de los ciudadanos y ciudadanas interculturales, propiciando su empoderamiento y a la vez genera capacidades para transformar las sociedades a las que pertenecen (URACCAN, 2014).

El modelo pedagógico se articula a partir de los fundamentos teóricos que ofrecen las pedagogías, articulado con el conocimiento tradicional ancestral (URACCAN, 2008). Reconoce y asume que lo pueblos indígenas, son portadores milenarios de conocimiento y saberes, que sus culturas enriquecen la cultura global de Latinoamérica. Que son de gran valía los valores éticos, humanos y espirituales que los pueblos trasmiten. Que la cultura y la relación que los pueblos asumen con el medio ambiente - su cosmovisión y cosmogonía- son fuentes inagotables y constituyen una energía revitalizadora y sustentable de saberes y conocimientos, que pueden ser fuentes teóricas y prácticas científicas para el autodesarrollo en el marco de una nueva relación horizontal con el mundo.

\section{Elementos metodológicos de la Universidad Comunitaria Intercultural}

Según el Proyecto Educativo Institucional (URACAN, 2008), la URACCAN se caracteriza por ser una organización académica regida por los siguientes elementos

\section{Metodológicos:}

1. El Papel que juega la comunidad en la universidad, los sabios, sabias, ancianos, ancianas y autoridades tradicionales y no tradicionales en los procesos educativos.

2. La búsqueda de nuevos paradigmas en los enfoques pedagógicos y metodológicos para la generación de conocimientos para el buen vivir de los pueblos.

3. La espiritualidad y el pensamiento mágico religioso.

4. La interculturalidad, la educación intercultural, la educación bilingüe.

5. Enfoque de género.

6. El papel central de la investigación y la innovación.

7. La articulación entre la teoría y la práctica.

8. Educación para toda la vida.

Este estudio resalta el que la URACCAN mantiene el principio de la relación teoría y práctica en los procesos de enseñanza aprendizaje, lo cual permite relacionar al sujeto en formación con la realidad de su entorno y de sus semejantes, los hace sensibles a las necesidades sociales y promueve el desarrollo del sentido del emprendimiento y la innovación en la solución de problemas de la sociedad (URACCAN, 2008).

\section{Infraestructura y tecnología}

La infraestructura tecnológica adecuada para fortalecer los procesos de enseñanza y aprendizaje. Asimismo, la Universidad reconoce la necesidad de seguir fortaleciendo las capacidades tecnológicas en los territorios, capacidades del personal docente y administrativo. En este sentido se desataca que el establecimiento del Laboratorio de Ciencias Naturales en el Recinto Nueva Guinea se dio en enero de 2011. 
Medio Ambiente y Recursos Naturales

\section{Materiales y métodos}

La investigación se realizó en el recinto URACCAN Nueva Guinea, RACCS, entre noviembre y diciembre de 2017. Se condujo desde una perspectiva mixta con predominancia del enfoque cuantitativo de tipo descriptiva, no experimental, de corte transversal.

La población fue de 268 estudiantes y 16 docentes de 6 carreras seleccionadas en las que sus currículos contemplan el uso de los Laboratorios de Ciencias Naturales. La muestra de estudiantes fue de 71. Se calculó con el software STATTM considerando un nivel de confianza del 95\%, un porcentaje esperado del $50 \%$ y un error máximo aceptable del 10\%. Para obtener la cantidad de estudiantes por carrera y género garantizando la representatividad de estas, se utilizó un muestreo estratificado, tomando los dos años superiores de las carreras incluidas. Se tomaron como parte de la población en estudio a los docentes que imparten clases en las carreras seleccionadas, específicamente de las asignaturas cuyos programas demandan el uso del Laboratorio de Ciencias Naturales. Se tomó la totalidad de ellos.

Otro elemento importante a destacar es el aporte que del responsable del laboratorio de Ciencias Naturales, la revisión de informes anuales del área en el período 2011-2017, así como los currículos, programas y syllabus de asignaturas vinculadas al uso del laboratorio.

Al tener una población representada por diversos estratos, surgió la necesidad de usar diversas técnicas e instrumentos, entre ellos: encuesta para docentes y estudiantes, la cual se operativizó a través de una guía de encuesta, entrevista para el responsable del Laboratorio, revisión documental para currículos, programas, syllabus, informes anuales y reglamento de laboratorio, utilizando las respectivas guías previamente elaboradas.

Las variables se midieron a través de proposiciones mediante una escala Likert, evaluadas con una puntuación del $1 \mathrm{al}$ 5, luego se sumó las puntuaciones por cada variable y para ponderar el 100\% y el porcentaje obtenido se tomó como referencia para su análisis, según los siguientes valores:

- 90-100\%: la variable medida fue considerada excelente.

- 80-89\%: la variable medida fue considerada muy buena.

- 70-79\%: la variable medida fue considerada buena.

- 6o-69\%: la variable medida fue considerada regular.

- Menor al 60\%: la variable medida fue considerada deficiente.

Para evaluar la incidencia de los laboratorios de Ciencias Naturales en el proceso de enseñanza - aprendizaje, se promediaron los porcentajes obtenidos en las variables evaluadas y a partir de ello se analizó la categoría incidencia en base a la escala indicada anteriormente. También se reforzó el análisis con los aspectos recopilados con las entrevistas y revisión documental. Para el establecimiento de las variables que permitieran medir la incidencia de las prácticas del Laboratorio de Ciencias Naturales en el aprendizaje, se adaptaron 4 categorías (variables) de Weyrauch, V. (2012), estas fueron:

Pertinencia de las prácticas: entendida como la correspondencia de las prácticas con las prioridades, necesidades y competencias establecidas en los currículos, programas de asignaturas, en esta se tomaron como subvariables: congruencia/coherencia, propósitos, tipos de prácticas, carreras, rol de los laboratorios

Calidad de las prácticas: La calidad se define desde la efectividad como la capacidad de lograr el cumplimiento de los objetivos del Laboratorio de Ciencias Naturales en condiciones habituales y desde la eficacia como la medida en que las prácticas alcanzan los objetivos propuestos, se tomaron como subvariables: capacidad operativa, asistencia/apoyo a las prácticas, contribución de las prácticas en el Laboratorio de Ciencias Naturales al aprendizaje de los estudiantes y orientación de estas. 
Impacto de las prácticas: entendido como los cambios positivos o negativos derivados de las prácticas, directa e indirectamente, intencional y no intencionalmente. Se abordaron las subvariables: actitud frente a las prácticas y fortalezas.

Sostenibilidad de las prácticas: entendida como la probabilidad de que los aprendizajes obtenidos en las prácticas continúen después de la misma. Las subvariables evaluadas: aplicación del conocimiento adquirido en las prácticas a la vida cotidiana y utilidad de las prácticas de laboratorio.

La información proveniente de las encuestas aplicadas a estudiantes y docentes se procesó haciendo uso del SPSS, en este se realizó la exploración de datos para la generación de tablas y gráficas, así como los análisis de parámetros de estadística descriptiva e inferencial.

\section{Resultados y discusión}

\section{Características generales de la población participante en el estudio}

La participación del estudiantado en el estudio estuvo representada por el $50 \%$ de hombres e igual porcentaje para las mujeres, en el caso de los docentes el $69 \%$ fueron hombres y el $31 \%$ mujeres.

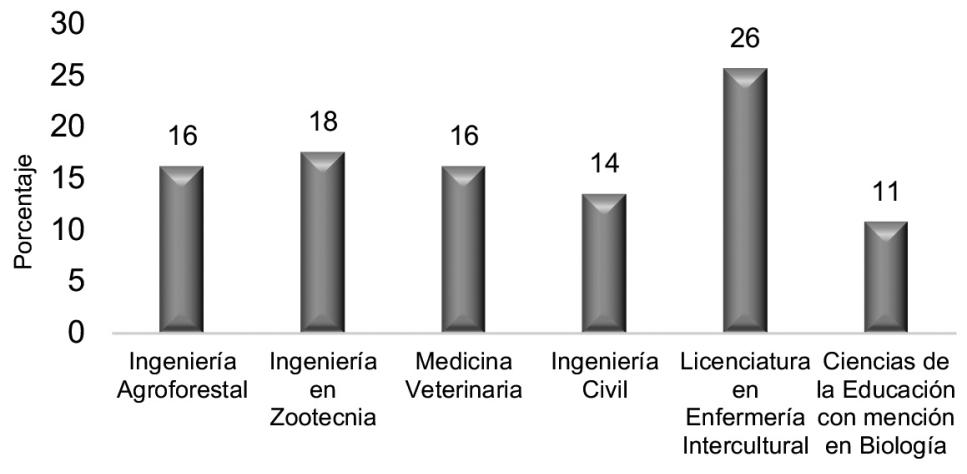

Figura No. 1. Docentes y estudiantes que participaron en el estudio.

Se consideraron las carreras que desde su dinámica, desarrollo y diseños curriculares plantean el uso del Laboratorio de Ciencias Naturales, en la figura No. 1 se representa, así como la cantidad de docentes y estudiantes que participaron en el estudio.

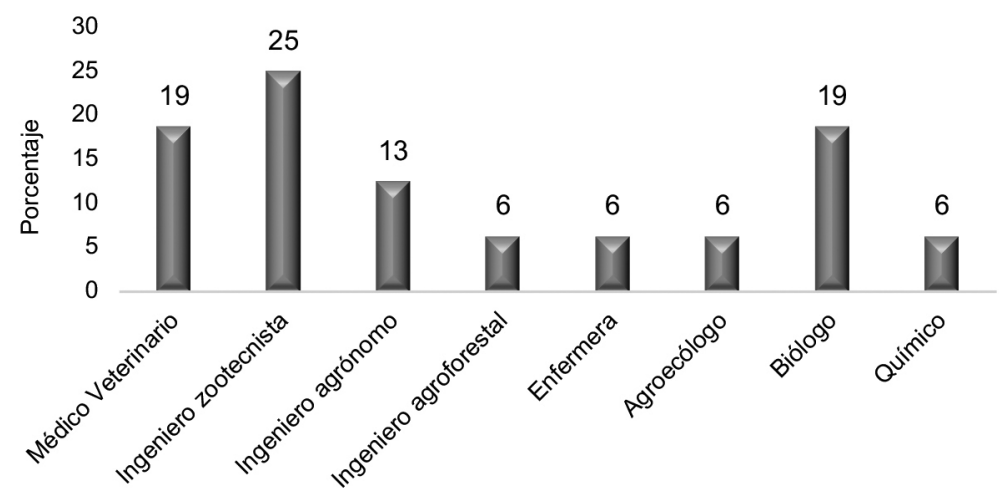

Figura No. 2. Profesiones de las y los docentes participantes en el estudio.

La figura No. 2 muestra la distribución de los docentes por profesión, la mayoría son ingenieros, esto es lógico, pues el laboratorio de Ciencias Naturales son por su propia naturaleza espacios de apoyo a estas 
profesiones. La mayoría de los docentes que fueron parte de esta investigación tienen nivel académico de licenciatura (ingenieros/as y licenciados/as), se debe aclarar que el $24 \%$ de estos están en proceso de culminación de maestría.

\section{Prácticas y servicios de los laboratorios de Ciencias Naturales}

Las carreras que hacen mayor uso de las diferentes prácticas y servicios que se orientan desde el Laboratorio de Ciencias Naturales del recinto, son Ingeniería en Zootecnia y Ciencias de la Educación con mención en Biología (Figura No. 3).

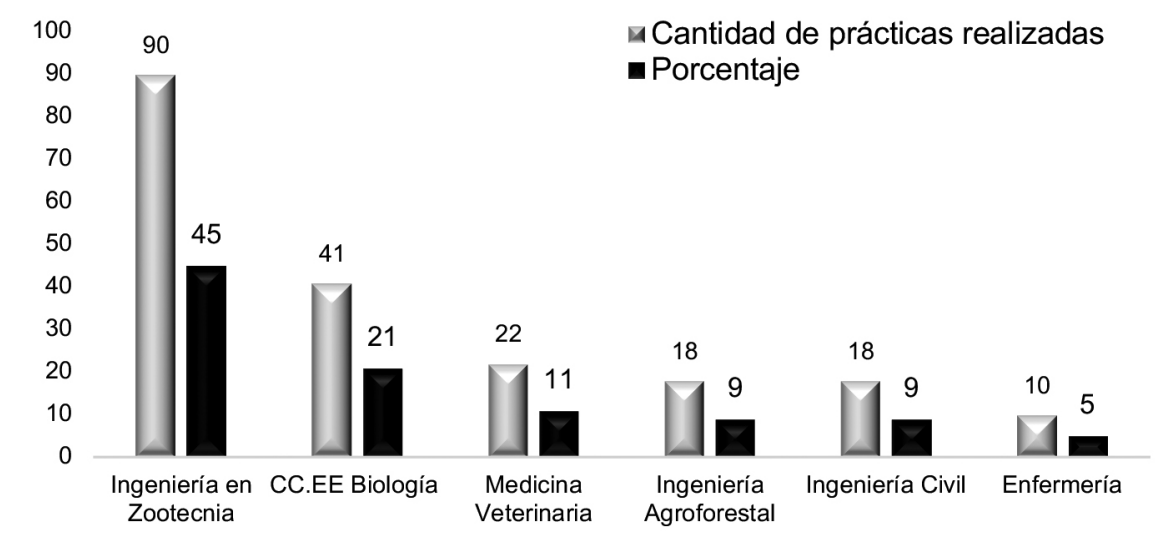

Figura No. 3. Carreras que han hecho uso del Laboratorio de Ciencias Naturales en el periodo 2011-2017.

Se destaca como un logro el hecho que desde 2011, fecha en que inicia actividades el Laboratorio de Ciencias Naturales a 2017, se han desarrollado 199 prácticas en 35 diferentes asignaturas con la participación de 5,539 estudiantes (2,145 mujeres) y 78 docentes (15 mujeres) para un total de 22,468 horas de prácticas de laboratorio. De las 199, el 80\% son profesionalizantes y el restante son básicas, estas concentradas en al menos 30 temáticas diferentes.

En los currículos y programas de asignaturas no se declara de forma explícita los tipos de prácticas requeridas, estas quedan a consideración del/la docente. Hay programas en los que se detallan las actividades prácticas como es el caso de técnicas de laboratorios, zoología y otras. En los syllabus se declara específicamente las actividades de laboratorios, esto a criterio del docente.

\section{Congruencia/coherencia de las prácticas en los laboratorios}

Como se aprecia en la figura No. 4, existe una percepción positiva de estudiantes y docentes respecto a la congruencia de las prácticas que se implementan en el laboratorio, solo una minoría considera que no son congruentes. Esta percepción positiva patenta el trabajo que se está haciendo en dirigir las prácticas a las necesidades de formación del estudiantado.

El responsable de laboratorio conforma que las prácticas cumplen las expectativas de los programas académicos y están orientadas a darle salida a alguna o varias temáticas relacionadas a las necesidades de formación del estudiantado. No obstante, estas se ven limitadas a los requerimientos (materiales, equipos y reactivos) disponibles.

Referido a la pertinencia del instrumental e insumos disponibles en el laboratorio de Ciencias Naturales, de la figura No. 5 se destaca una valoración favorable por parte de los docentes. La insatisfacción mostrada por el estudiantado obedece a la poca disponibilidad de reactivos y equipos para prácticas específicas. La percepción favorable por docentes y estudiantes concuerda con Colado, (s.f.), quien plantea que toda tarea 
práctica implica una correcta percepción de los objetos, sus cualidades y sus relaciones y esto se logra con el uso de los laboratorios y sus recursos a la disposición del estudiantado.

En los currículos no se detalla con precisión los requerimientos de insumos e instrumentos de laboratorio para las prácticas, pero si en la mayoría de los programas y syllabus. Lo referido a reactivos tampoco queda claro, este aspecto es mencionado en el capítulo de recursos didácticos necesarios de los programas, consideramos que se debería designar un capítulo exclusivo para esto.

Tabla 1. Rol del Laboratorio de Ciencias Naturales en URACCAN Nueva Guinea.

\begin{tabular}{lcc}
\hline Categorías (porcentaje) & Estudiantes & Docentes \\
\hline $\begin{array}{l}\text { Desarrollar habilidades y destrezas técnicas en el estudiantado } \\
\text { Fortalecer los conocimientos teóricos mediante la experimentación }\end{array}$ & 12 & 50 \\
$\begin{array}{l}\text { Promover la relación entre teoría y práctica para la aplicación de } \\
\text { conocimientos }\end{array}$ & 11 & 38 \\
$\begin{array}{l}\text { Generar conocimientos aplicados a realidades específicas del campo de } \\
\text { formación del estudiantado }\end{array}$ & 5 & 6 \\
$\begin{array}{l}\text { Desarrollar habilidades y destrezas técnicas y promover la relación entre } \\
\text { teoría y práctica }\end{array}$ & 3 & 0 \\
$\begin{array}{l}\text { Desarrollar habilidades y destrezas técnicas y generar conocimientos } \\
\text { aplicados a realidades específicas }\end{array}$ & 1 & 0 \\
$\begin{array}{l}\text { Desarrollar habilidades y destrezas técnicas y fortalecer los } \\
\text { conocimientos teóricos mediante la experimentación }\end{array}$ & 41 & 0 \\
Ninguna de las anteriores & 1 & 0 \\
\hline
\end{tabular}

En el cuadro No. 1 se visualiza la percepción de estudiantes y docentes sobre el rol que debe jugar el laboratorio de Ciencias Naturales, estos coinciden en que éste debe enfocarse en el desarrollo de habilidades y destrezas y promover la experimentación. Por otro lado, desde la perspectiva del responsable de los laboratorios, básicamente el rol de estos es servir de puente que fortalezca conocimientos, desarrolle hábitos, habilidades, destrezas y enseñe técnicas específicas vinculadas a temáticas del campo de formación del estudiantado. La disponibilidad de estos espacios también permite demostrar supuestos teóricos mediante la puesta en práctica que conllevan a adquirir conocimientos sólidos permanentes, son espacios donde se logra demostrar que se aprende mejor desde la práctica.

En relación a los roles que cumplen los Laboratorios de Ciencias Naturales, López y Tamayo (2012), afirman que estos brindan a los estudiantes la posibilidad de entender cómo se construye el conocimiento dentro de una comunidad científica, cómo trabajan los científicos, cómo llegan a acuerdos y cómo reconocen desacuerdos, qué valores mueven la ciencia, cómo se relaciona la ciencia con la sociedad y con la cultura. Sobre esa misma lógica, Cardona (2013) lo sustenta planteando que el objetivo principal de la práctica de laboratorio es facilitar que los alumnos lleven a cabo sus propias investigaciones, se contribuye a desarrollar su comprensión sobre la naturaleza de la ciencia y su reflexión sobre el propio aprendizaje personal.

Como aporte al rol del laboratorio de Ciencias Naturales, Woolnough y Allsop citado por Barberá y Valdés (1996) sostienen que, los objetivos propios de estos son proporcionar experiencia directa sobre fenómenos, permitir contrastar la abstracción científica ya establecida con la realidad que pretende describir, desarrollar competencias técnicas y desarrollar el razonamiento práctico. Por otra lado Kirschner (1992), refiere que el trabajo práctico se debe utilizar para enseñar y aprender la estructura sintáctica de una disciplina, más que la estructura sustantiva y plantea tres razones o motivos válidos para ello: desarrollar destrezas específicas a través de ejercicios, aprender el "enfoque académico" a través de los trabajos prácticos como investigaciones, de modo que el estudiante se involucre en la resolución de problemas como lo hace un científico y tener experiencias con fenómenos. 


\section{Evaluación de la incidencia del laboratorio de Ciencias Naturales desde la percepción de la pertinencia}

La pertinencia de las prácticas fue considerada muy buena por estudiantes y docentes (80 y $87 \%$, respectivamente), es notorio resaltar que a pesar del poco tiempo de funcionamiento del laboratorio y algunas limitantes por superar, la pertinencia de los procesos que en estos se desarrollan ha sido un elemento característico en todas las carreras que hacen uso de estos espacios.

Lugo (2006) plantea que la pertinencia de un laboratorio debe concebirse desde la definición de este como un espacio equipado con diversos instrumentos de medición, donde se realizan experimentos o investigaciones diversas, según la rama de la ciencia a la que se enfoque. Para que estos espacios se correspondan tanto al ámbito académico como industrial deben responder a múltiples propósitos sea para la enseñanza, para la investigación o para la certificación de la industria.

Calidad en los procesos y/o actividades desarrolladas en el laboratorio de Ciencias Naturales

\section{Efectividad: Procesos definidos para la planificación y ejecución de prácticas}

El Laboratorio de Ciencias Naturales dispone de un sistema organizacional derivado de las políticas de gestión institucional. Cuenta con procesos definidos para la administración efectiva de sus recursos que permiten la planificación y ejecución de las prácticas que derivan del Reglamento de uso y funcionamiento del laboratorio de Ciencias Naturales. Este reglamento no ha sido aprobado por el Consejo Universitario de URACCAN (CUU), no obstante, ha servido de guía para orientar los procesos operativos en este espacio.

Del reglamento se derivan los formatos de solicitud para espacios, materiales, equipos y reactivos químicos que los docentes y estudiantes requieren para sus prácticas. Se cuenta con formatos digitales de pre-planificación de prácticas donde los docentes declaran los requerimientos por semestre para el desarrollo de sus prácticas. En relación a los formatos para guías de laboratorio que se emplean, solamente se orienta su elaboración desde el reglamento, no obstante, existe un documento que describe la estructura de la misma, así como directrices específicas para la elaboración de reportes de laboratorio como insumo de evaluación de los aprendizajes.

Estudiantes y docentes opinaron de manera positiva sobre los procedimientos definidos para la planificación y ejecución de las prácticas (Figura No. 6), esta apreciación se corresponde con lo expresado por el responsable del laboratorio.

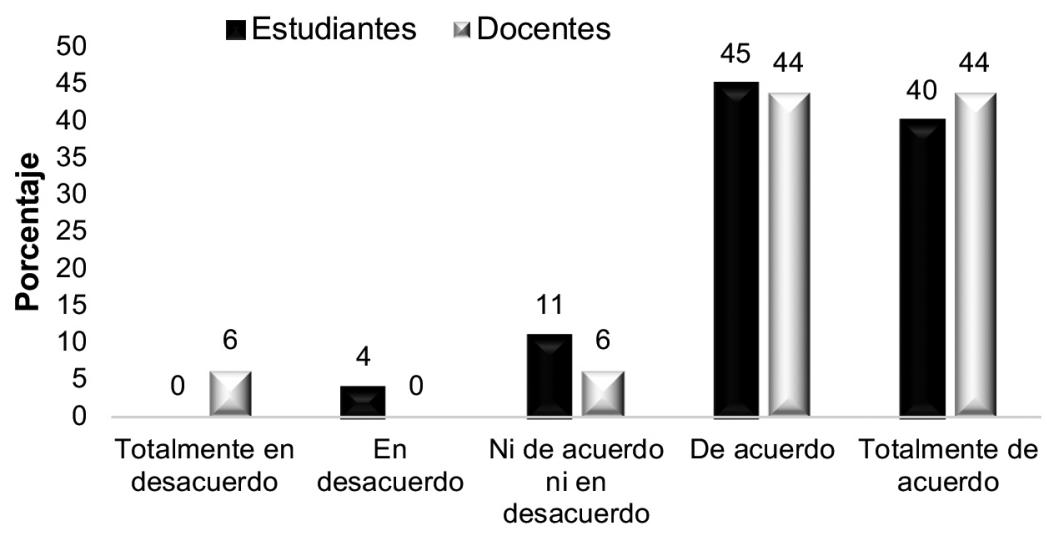

Figura No. 6. Apreciación de estudiantes y docentes respecto a la planificación y ejecución de las prácticas. 


\section{Disponibilidad de recursos en el Laboratorio de Ciencias Naturales}

En relación a esto, el responsable del laboratorio indicó que se dispone de los materiales básicos, algunos equipos y reactivos que permiten implementar prácticas específicas que favorecen el aprendizaje enfocado al campo de formación del estudiantado. No obstante, los usuarios directos (estudiantes y docentes) manifestaron apreciaciones diferentes (Figura No. 7). El estudiantado refiere mayor desacuerdo sobre las capacidades instaladas mientras que los docentes lo respaldan de manera positiva.

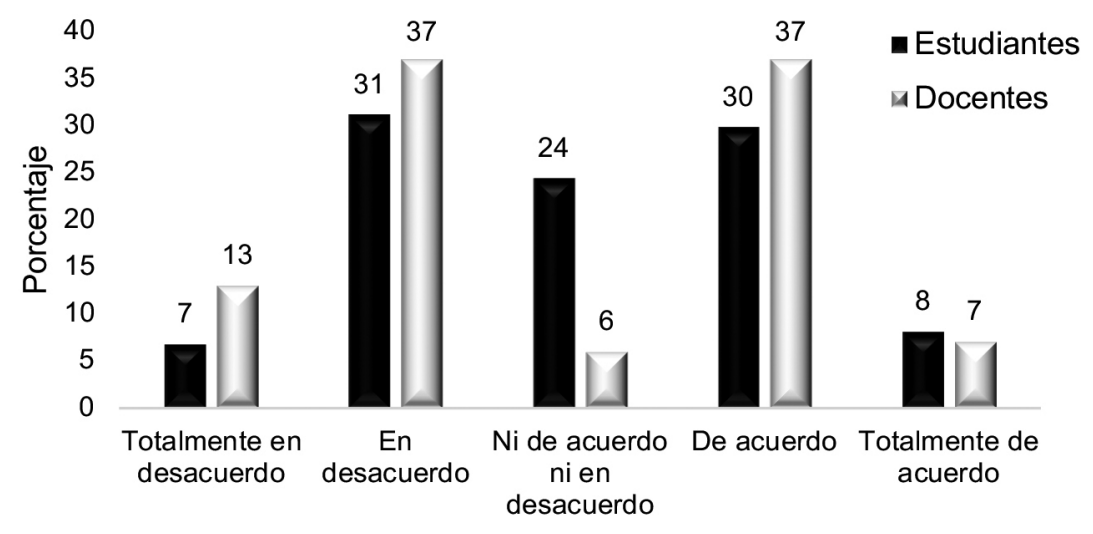

Figura No. 7. Percepción de estudiantes y docentes sobre disponibilidad de recursos en el laboratorio.

Es importante destacar que el estudiantado emite su juicio sobre la disponibilidad en relación a su campo de formación y es razonable hasta cierto punto; sin embargo, la prioridad del laboratorio es brindar conocimientos generales que permitan desarrollar habilidades y capacidades en ciertas temáticas para afrontar con éxito las particularidades de su campo ocupacional.

\section{Asistencia/apoyo en las prácticas de laboratorio}

La asistencia a las prácticas de laboratorio se da en dos vías, de responsable a estudiantes y docentes previo a realización de la práctica de laboratorio; y de docentes a estudiantes cuando se ejecuta dicha práctica. La asistencia se centra en actividades como: préstamos de equipos y dispositivos de medición de campo, acompañamiento en prácticas que algunos docentes orientan fuera de su horario de clases establecido, recepción de solicitud de materiales, equipos y reactivos, asistencia y/o capacitación en uso y manejo de equipos y dispositivos de campo, asistencia técnica en temáticas específicas, facilitación de información para elaboración de guías de prácticas y directrices para elaboración de reportes. Lo anterior corresponde con lo encontrado en la revisión documental, puesto que las actividades de asistencia y apoyo descritas están orientadas a la capacitación en usos y manejo de equipos, estudio y aplicación de normas de seguridad, recepción de solicitudes para prácticas (espacios, materiales, reactivos y equipos) y asistencia durante el desarrollo de las prácticas desarrolladas.

Por su parte los estudiantes, se refirieron a la utilidad del acompañamiento brindado por el responsable y docentes, encontrándose una apreciación positiva de 80 a 89 \% de satisfacción.

\section{Estadística del uso del laboratorio de Ciencias Naturales}

Las estadísticas presentadas en esta investigación cubren el período 2011-2017. No obstante, en el 2011-2012 solamente se atendían tres carreras (Zootecnia, Agroforestal y Biología) y fue a partir del 2014 que se amplió la oferta incorporándose ingeniería civil, medicina veterinaria, enfermería intercultural. Se contabilizaron 199 prácticas en el período referido con la participación de 5539 estudiantes (2145 mujeres) y 78 docentes (15 mujeres). 
La cantidad de prácticas realizadas se deben a una mayor disponibilidad y compromiso de los docentes en la planificación y ejecución de prácticas, adicionalmente, la importancia de poseer una formación científica-técnico y en algunos casos de especialización en el campo práctico.

A medida que se fortalezcan las capacidades en cuanto a equipamiento, ampliación de infraestructura, habilitación de espacios y dotación de reactivos químicos se evidenciará mayor uso de las potencialidades del laboratorio en un aumento significativo de la cantidad de prácticas y sobre todo orientadas al perfil profesional de cada carrera.

En relación a la cantidad de asignaturas se contabilizó un total de 35, donde las temáticas abordadas más relevantes se clasificaron en básicas y profesionalizante o aplicaciones en el campo de formación y estuvieron enfocadas en familiarización con instrumental del laboratorio (Biología/Veterinario, Suelo y Agua), técnicas generales medición de masas y volúmenes, uso y manejo de microscopios, balanzas analíticas, entre otras; las del área profesionalizante se enfocaron en estimaciones de calidad de aguas naturales y tratadas, fertilidad de suelos, diagnóstico de parásitos internos y externos en ganado bovino, técnicas de cirugía menor y determinaciones microbiológicas.

La consideración de incidencia del laboratorio al aprendizaje es positiva, si se visualiza la cantidad de estudiantes que participaron en procesos de formación dentro de laboratorio como un aporte significativo al aprendizaje teórico en el aula, por otro lado, resulta que muchos docentes, sobre todo del sexo femenino, no están teniendo un involucramiento directo y efectivo en prácticas.

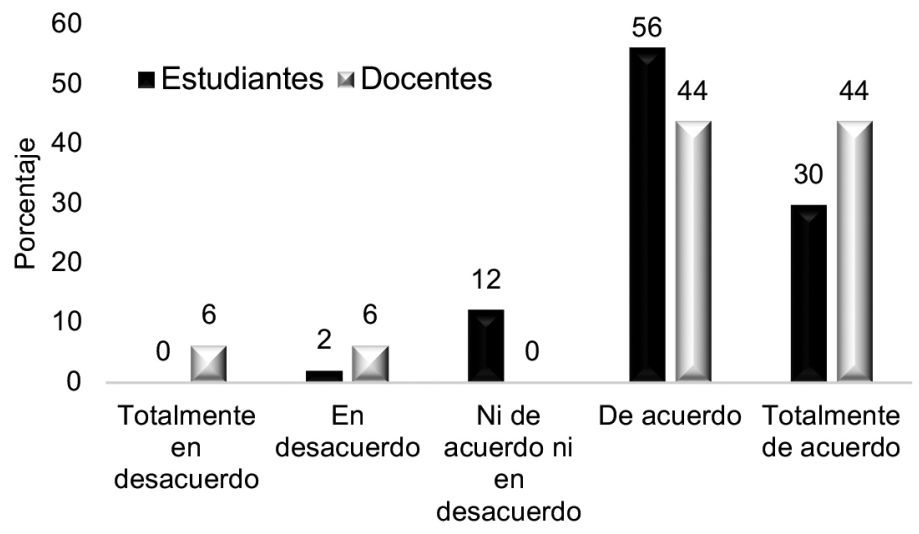

Figura No. 8. Consideración de docentes y estudiantes sobre disponibilidad de espacios para realizar sus prácticas.

Respecto a la apreciación de estudiantes y docentes en cuanto sobre la disponibilidad de espacios para el desarrollo de sus prácticas (ver figura No. 8) estos indicaron estar de acuerdo.

\section{Eficacia: Contribución al aprendizaje}

Para respaldar las afirmaciones anteriores, estudiantes y docentes coincidieron al reafirmar que las prácticas de laboratorio que realizaron les permitieron consolidar sus conocimientos generales tanto teóricos como profesionalizantes. Como lo muestra la figura No. 9. Es natural encontrar discrepancias entre ambas opiniones, pues el estudiantado generalmente adopta una posición pasiva cuando se trata de expresar su sentir respecto a procesos de aprendizajes.

En la figura No. 10 se observa una inclinación altamente satisfactoria respecto a la contribución de las prácticas de laboratorio que realizaron en el laboratorio de Ciencias Naturales para el aprendizaje del estudiantado que al ser evaluados, el nivel promedio de incidencia es del $90 \%$. 


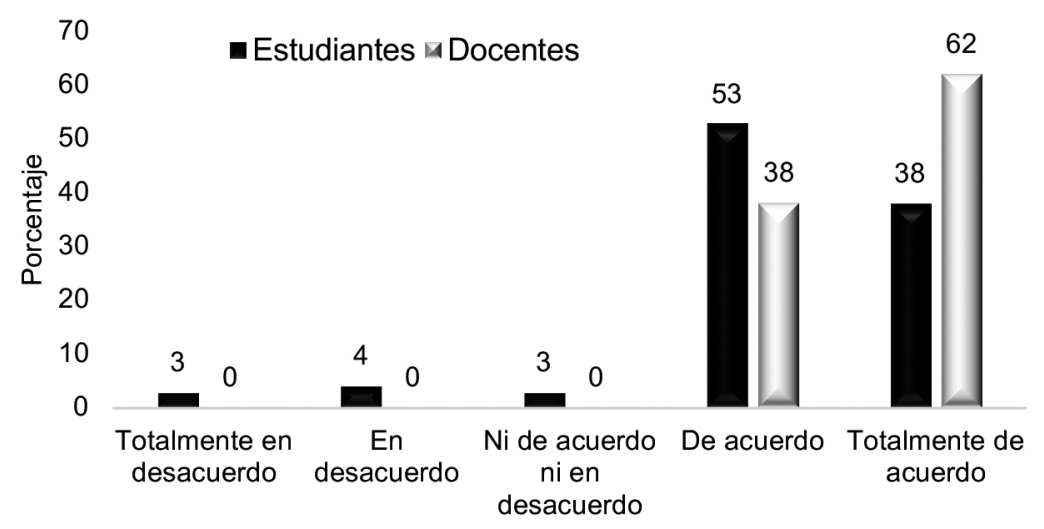

Figura No. 9. Consolidación del conocimiento a través de las prácticas de laboratorio.

Si bien es cierto que lo presentado tiene prevalencia positiva, es importante destacar otra apreciación del responsable del laboratorio, quien además de resaltar aspectos favorables indicando que dichas prácticas cumplen las expectativas de los programas académicos y están orientadas a darle salida a alguna o varias temáticas relacionada a las necesidades de formación del estudiantado, también remarca que muchas de estas prácticas se ven limitadas por falta de requerimientos (materiales, equipos y reactivos) disponibles, es decir, no se pueden ampliar a otras temáticas e incluso a otras asignaturas que demandan la realización de este tipo de actividades.

\section{Orientación de las prácticas de laboratorios}

El laboratorio dispone de un reglamento de uso y funcionamiento que intenta regular la buena administración de los recursos, la planificación y ejecución de las prácticas. El responsable del laboratorio refirió que en el reglamento del laboratorio están definidos dichos procesos. En relación a los elementos que deben tener las guías de prácticas de laboratorio indicó que no están precisados, pero los docentes deben proporcionar las guías de prácticas al inicio de sus cursos académicos y con antelación al estudiantado. El reglamento no cuenta con un acápite donde se establezcan aplicación de sanciones por incidencia en diferentes tipos de faltas dentro del laboratorio ni al momento del desarrollo de las prácticas. Las medidas de seguridad están bien establecidas.

Para tener certeza de las afirmaciones anteriores de manera objetiva se logró conocer la percepción de docentes y estudiantes sobre la calidad y metodología utilizada en la implementación de las prácticas en los Laboratorios, los resultados obtenidos se presentan en el cuadro No. 6 (indica que los docentes no llevan estructurada la guía de laboratorio)

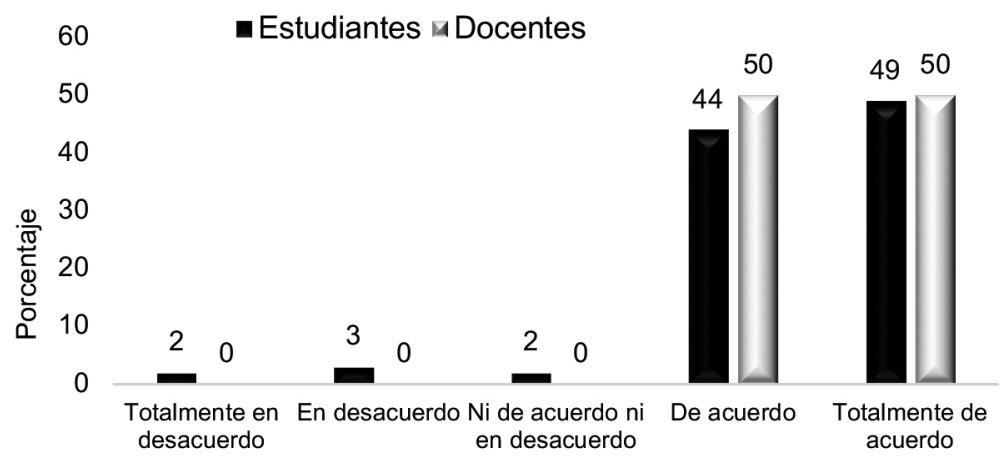

Figura No. 10. Contribución de las prácticas en los Laboratorios de Ciencias Naturales al aprendizaje.

Los docentes y estudiantes opinaron en relación a la existencia o establecimiento de normas claras que favorecen o no el desarrollo de las actividades en el laboratorio de Ciencias Naturales. Tal como se aprecia en la figura No. 11 ambos sujetos reafirman lo planteado por el responsable del laboratorio y con 
lo encontrado en la revisión documental. El nivel de incidencia del laboratorio en el proceso de enseñanza-aprendizaje en la escala establecida para valorar dicho indicador (90-100 \%) en esta investigación es excelente.

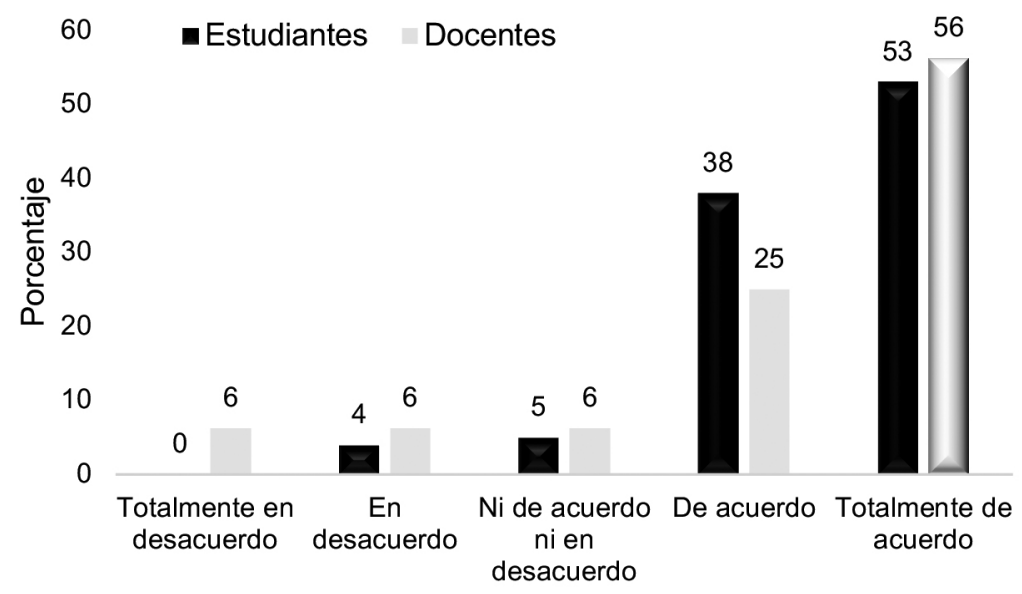

Figura No. 11. Existencia de mecanismos y documentos normativos que regulen el buen desarrollo de las prácticas de laboratorio.

\section{Evaluación de la incidencia del laboratorio de Ciencias Naturales en la educación desde la percepción de la calidad}

La variable calidad fue considerada buena y muy buena por estudiantes y docentes (79 y $82 \%$, respectivamente) según las escalas de evaluación definidas en este estudio. Este resultado denota que existe calidad en los procesos y/o actividades (prácticas de laboratorio) desarrolladas en el Laboratorio de Ciencias Naturales y aportan al proceso de aprendizaje significativo del estudiantado y docentes.

Respecto a la incidencia de los laboratorios en los procesos de enseñanza aprendizaje y la calidad de las prácticas, Urrea et al. (2013) y Colado (s.f.) señalan que las prácticas de calidad requieren de tiempo adicional para su planificación, pero que su vez constituyen una fuente importante en la formación del individuo, que dejará una huella imperecedera en él y que, en gran medida, será decisiva en su ulterior desarrollo, así como en su proyección ante la vida y su concepción del mundo.

\section{Incidencia y potencialidades del laboratorio de Ciencias Naturales en la educación}

\section{Impacto de las prácticas de laboratorios}

Para valorar la apreciación del impacto que ha generado el desarrollo de prácticas de laboratorio como aporte significativo complementario para entender la teoría de las temáticas establecidas en los diferentes programas académicos de las carreras consideradas en este estudio, se consultó a docentes y estudiantes sobre su actitud y participación desde el enfoque perceptivo positivo, indiferente y pasivo.

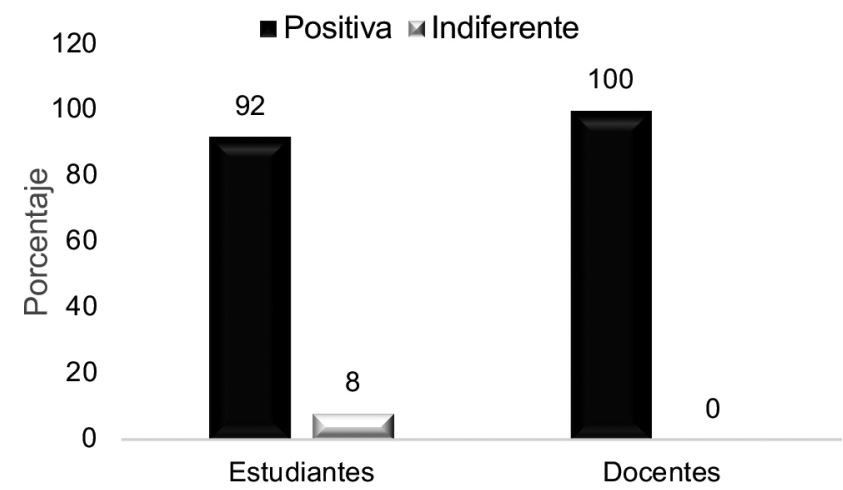

Figura No. 12. Actitud de estudiantes y docentes frente a las prácticas de laboratorio. 
En relación a la actitud frente a las prácticas de laboratorio, el proceso de autoevaluación de docentes y estudiantes (Figura No. 13) es altamente positiva donde la escala de incidencia tomó valores de 90 a $100 \%$ indicando balance positivo en ambas apreciaciones y un nivel de satisfacción excelente.

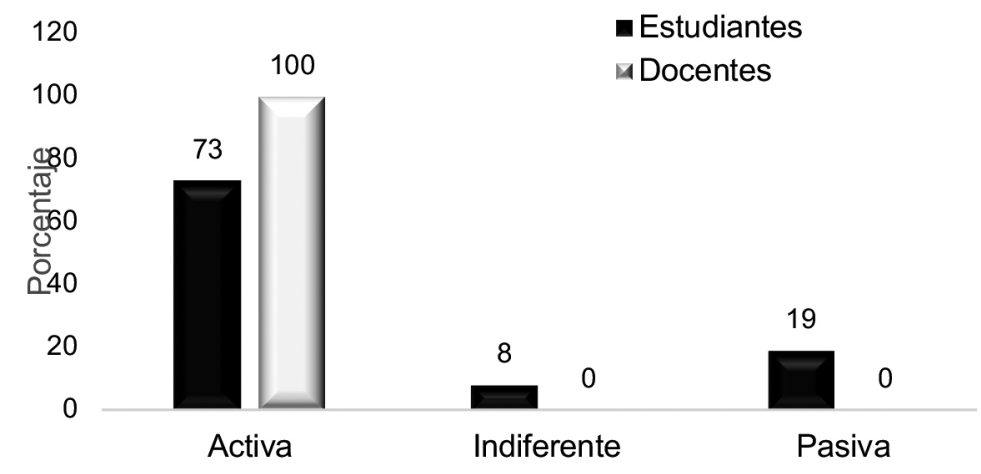

Figura No. 13. Participación de estudiantes y docentes en las prácticas de laboratorio.

Respecto a la valoración de docentes y estudiantes en relación a la participación en las prácticas de laboratorio que realizaron, se evidencia mayores apreciaciones. Los docentes sostienen que fueron activos sus involucramientos;, no obstante, los estudiantes sólo el 73 \% apuntó a esta apreciación, distribuyéndose en pasiva e indiferente un $27 \%$ del total consultado (Figura No. 13). Aun así, con estos resultados se considera satisfactoria en una escala buena, no obstante, hay correspondencia con la cantidad de estudiantes que opinaron en relación a la infraestructura de los laboratorio (Figura No. 23), probablemente la actitud y participación del estudiantado tenga que ver con el grado de motivación y comodidad que existe en el laboratorio, siendo estos elementos que no favorezcan positivamente el desarrollo de sus prácticas en función de la infraestructura, pero no de la adquisición de conocimientos.

\section{Fortalezas de las prácticas, espacios y recursos existentes}

Las fortalezas en relación a disposición de infraestructura, recursos e insumos con las que se cuentan, este destaca:

1. Contar con estos espacios de aprendizaje es ya una fortaleza, puesto que son muchas las asignaturas que contemplan temáticas o supuestos teóricos que es necesario sean llevadas a la práctica para poder asimilar significativamente el contenido.

2. Se dispone de los materiales básicos, algunos equipos y reactivos que permiten implementar algunas prácticas específicas que favorecen el aprendizaje enfocado al campo de formación del estudiantado.

3. La infraestructura, componentes, disponibilidad y orden de los mismos es en sí una forma de aprender en relación a las medidas organizativas y de dirección de un laboratorio.

4. El laboratorio de Ciencias Naturales es un medio de consolidación de los conocimientos que se desarrolla en el aula de forma teórica.

5. Ampliación de metodologías analíticas orientadas al sector agropecuario (diagnóstico veterinario) donde los beneficiarios indirectos son los estudiantes.

Por otro lado, desde la revisión documental el responsable menciona otros logros adquiridos vinculados directamente con las prácticas de laboratorio que se describen enseguida:

1. Asistencia técnica permanente a docentes y estudiantes.

2. Mayor cantidad de prácticas realizadas.

3. Convenio INTA-URACAN, Nueva Guinea.

4. Ampliación de metodologías analíticas para el análisis de rutinas de suelo. 
5. Mayor involucramiento y participación en el área del laboratorio por docentes y estudiantes en experiencias prácticas en este.

6. Adquisición de materiales quirúrgicos para la carrera de medicina veterinaria.

7. Adquisición de reactivos químicos para apoyar la docencia y el establecimiento de parámetros físicos y químicos en matrices suelo y agua.

8. Instalado sistema de abastecimiento de agua potable en el laboratorio.

9. Mayor involucramiento de docentes horarios en prácticas de laboratorio.

10. Articulación entre coordinadores de áreas académicas y laboratorio para motivar a docentes a desarrollar prácticas.

11. Prácticas de laboratorio enfocadas a temáticas vinculadas directamente con el perfil ocupacional del estudiantado.

12. Revisión y mantenimiento preventivo y correctivo de equipos de laboratorio.

Es importante señalar que desde los currículos no se detallan los requerimientos de cada carrera en cuanto las prácticas en el laboratorio de Ciencias Naturales. No obstante, en la mayoría de los programas y syllabus donde se consuma el Currículo, se mencionan los requerimientos de equipos y materiales principalmente, lo referido a reactivos, este aspecto es mencionado en el capítulo de recursos didácticos necesarios.

1. Si bien es cierto el responsable menciona algunas fortalezas, también expresa algunas limitantes que no han permitido ampliar las prácticas y por tanto han desfavorecido en alguna medida el aprendizaje significativo en el estudiantado, entre ellas mencionó:

2. Carencia de algunos materiales, equipos y reactivos químicos específicos para el desarrollo de técnicas analíticas que es necesario desarrollar en ciertas temáticas de asignaturas como química orgánica, fisiología vegetal, genética, botánica, entre otras.

3. La cantidad de materiales y algunos equipos existentes no son suficientes para que los estudiantes realicen los experimentos de manera individual, esto implica que algunos no tienen la oportunidad de manipularlos y poner en práctica lo orientado de la manera adecuada.

4. En algunos cursos, se realizan pocas prácticas y en otros casos no se realizan, aun en asignaturas con temáticas que demandan el desarrollo de las mismas.

5. En algunos casos se evidencia que los docentes no elaboran una guía de práctica de laboratorio y solo orientan el desarrollo de los experimentos en el momento de la clase.

6. Docentes muestran poco interés por realizar prácticas de laboratorio

7. Aplicación no adecuada del reglamento de laboratorio.

\section{Evaluación de la incidencia del laboratorio de Ciencias Naturales en la educación desde la percepción del impacto}

La variable impacto fue considerada buena y muy buena por estudiantes y docentes ( 79 y $83 \%$, respectivamente), es evidente que estas actividades han generado cambios de actitud positivos para la formación del estudiantado, mismo que les permitió tanto a docentes y estudiantes autoevaluarse y evaluar todos los procesos vinculados al quehacer del laboratorio y el impacto que se aprecia en mayores conocimientos, habilidades y capacidades de la comunidad de aprendizaje.

\section{Sostenibilidad: Utilidad de las prácticas de laboratorios}

Como lo muestra la figura No. 14, la mayoría de estudiantes y docentes concuerdan en que las prácticas que desarrollan en el laboratorio de Ciencias le son de utilidad para la realización de investigaciones, este aspecto es importante, pues esta es una de las premisas de estos espacios y permite la conexión con otras asignaturas que no precisamente requieren del uso de laboratorios, pero su desarrollo se beneficia por el hecho de que los estudiantes han adquirido habilidades y destrezas investigativas útiles a cualquier ámbito de la carrera. 


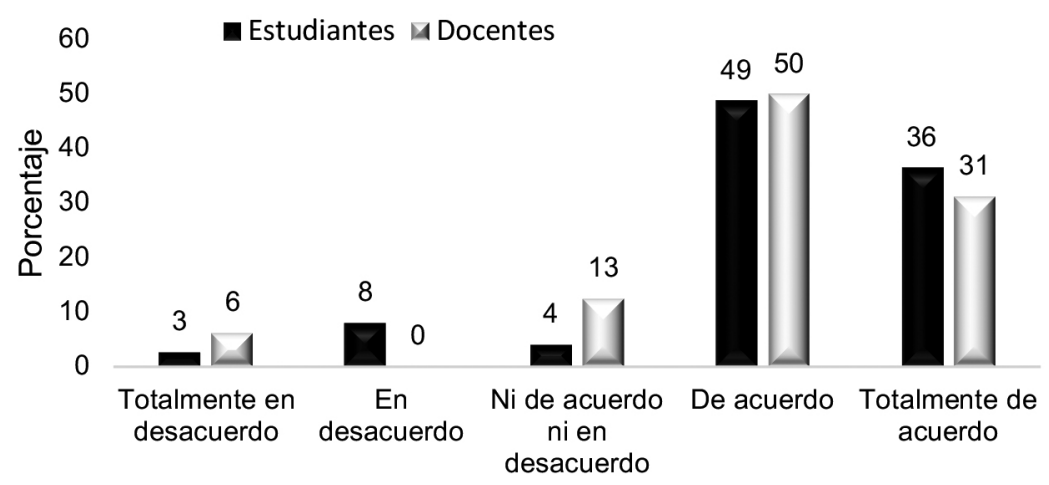

Figura No. 14. Utilidad de las prácticas en el Laboratorio de Ciencias Naturales para realizar investigaciones.

Salcedo et al. (2005), refuerzan lo anterior con la afirmación de que las prácticas de laboratorio juegan un papel primordial en la familiarización de los estudiantes con la metodología científica y por ende con sus capacidades investigativas.

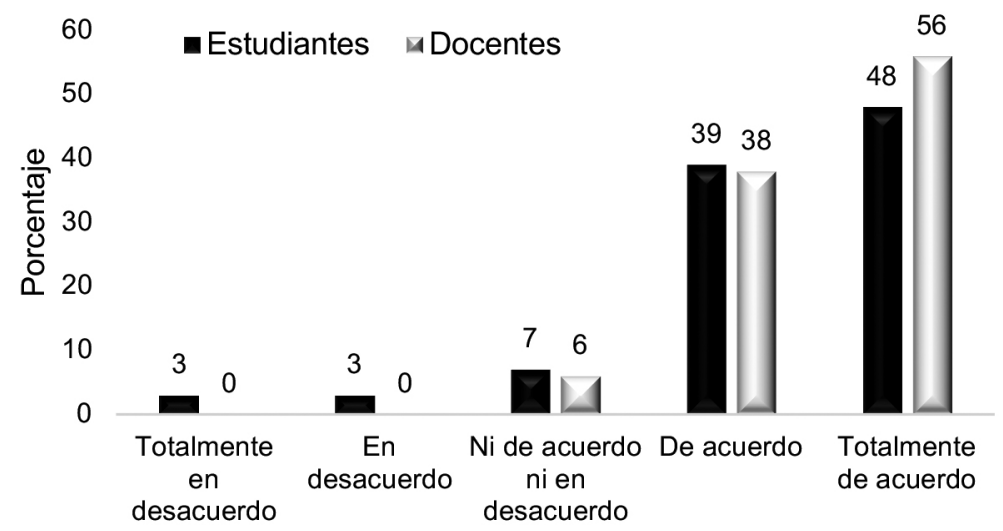

Figura No. 15. Utilidad de los aprendizajes adquiridos en las prácticas de laboratorio para el desempeño de la carrera.

Es importante resaltar la percepción positiva de estudiantes y docentes respecto a la utilidad de las prácticas realizadas en el laboratorio de Ciencias Naturales (figura No. 15), esto apoya la tesis de que los aprendizajes en los laboratorios deben ser coherentes con la práctica de la vida cotidiana y por ende útiles para los fines académicos que se persiguen y para desarrollar habilidades técnicas en los aprendientes.

A partir de la figura No. 16 se aprecia como aspecto importante a destacar la percepción de estudiantes y docentes sobre la utilidad de los aprendizajes para lograr en un efectivo desempeño de su carrera, en este particular, estudiantes y docentes coinciden en una apreciación positiva sobre que desde estos espacios académicos se está preparando al estudiantado para un mejor desempeño profesional.

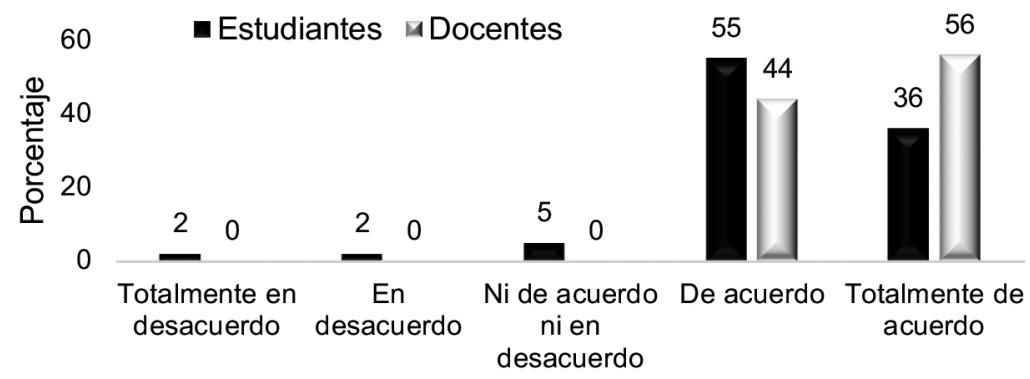

Figura No. 16. Utilidad técnica y académica de los conocimientos adquiridos en las prácticas de laboratorios. 


\section{Aplicación de las prácticas laboratorios}

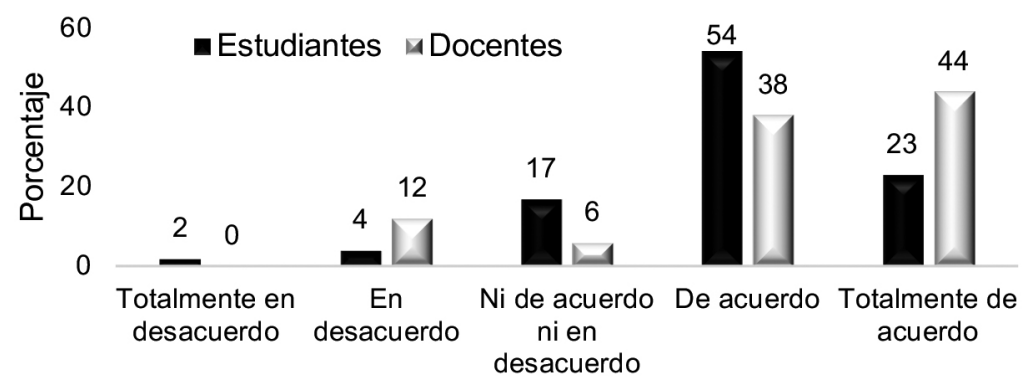

Figura No. 17. La experiencia práctica en los laboratorios propicia el desarrollo de habilidades aplicables a una variedad de asignaturas.

La figura No. 17 muestra que respecto a la aplicación de las prácticas de laboratorio a la vida cotidiana del estudiantado vista como la posibilidad de aplicar los conocimientos adquiridos a su realidad y a su entorno, la mayoría de estudiantes y docentes están de acuerdo que en efecto dicha aplicación inicia desde el hecho que las prácticas les permiten el desarrollo de habilidades aplicables a una variedad de asignaturas, es decir en el ámbito académico inmediato del estudiante ya se ve un efecto positivo, sobre ese mismo aspecto, estudiantes y docentes coinciden en que también contribuyen de forma significativa a desarrollar la comprensión sobre la naturaleza de la ciencia y su reflexión sobre su aprendizaje personal.

Siempre referido a la aplicación de las prácticas de laboratorios, hay una percepción positiva de estudiantes y docentes respecto a la visualización de los contenidos teóricos en las prácticas a las que ellos asisten, es decir se está dando la posibilidad de que las actividades desarrolladas en estos espacios realmente tengan un significado práctico/aplicable a contextos reales propios de la asignatura y de la carrera.

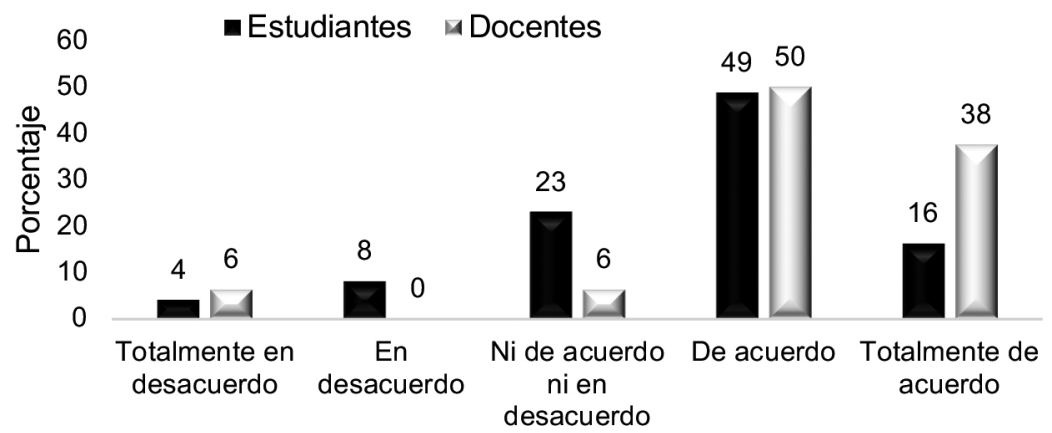

Figura No. 18. Visualización de los contenidos teóricos en las prácticas del Laboratorio de Ciencias Naturales.

\section{Evaluación de la incidencia del Laboratorio de Ciencias Naturales desde la percepción de la sostenibilidad}

La variable sostenibilidad fue considerada como muy buena por estudiantes y docentes ( 82 y $84 \%$, respectivamente), este resultado denota que las prácticas desarrolladas tienen una aplicabilidad y utilidad significativa tanto en la vida académica como en la vida cotidiana del estudiantado.

\begin{tabular}{|l|l|l|}
\hline \multicolumn{3}{|c|}{ Cuadro No. 2. Evaluación general de las variables } \\
\hline \multicolumn{1}{|c|}{ Variables } & \multicolumn{1}{|c|}{ Evaluación cuantitativa (\%) } & \multicolumn{1}{c|}{ Evaluación cualitativa } \\
\hline Pertinencia & 84 & Muy buena \\
\hline Calidad & 82 & Muy buena \\
\hline
\end{tabular}




\begin{tabular}{|l|l|l|}
\hline \multicolumn{3}{|c|}{ Cuadro No. 2. Evaluación general de las variables } \\
\hline \multicolumn{1}{|c|}{ Variables } & \multicolumn{1}{|c|}{ Evaluación cuantitativa (\%) } & \multicolumn{1}{c|}{ Evaluación cualitativa } \\
\hline Impacto & 81 & Muy buena \\
\hline Sostenibilidad & 83 & Muy buena \\
\hline Promedio & 82 & Muy buena \\
\hline
\end{tabular}

El $82 \%$ de promedio general refleja una incidencia muy buena del Laboratorio de Ciencias Naturales en el aprendizaje de los estudiantes que hacen uso de él. Este hecho se fortalece con lo establecido por Scoles y Patticini en Urrea et al. (2013) quienes concluyeron que la aplicación de prácticas de laboratorios como estrategia de aprendizaje viene a ser una opción frente al desarrollo de la enseñanza tradicional, pues resulta eficaz para un aprendizaje significativo, recomendando el empleo de métodos menos pasivos para el estudiante, ya que los mismos perciben el laboratorio como un lugar donde están activos. Por su parte Hodson (1994) remarca que innovar en las prácticas de laboratorio teniendo en cuenta lo que el alumno ya sabe juega un papel fundamental en lo que este aprende.

Por los propósitos de este estudio se resalta lo que establece URACCAN (2008) quien mantiene el principio de la relación teoría y práctica en los procesos de enseñanza aprendizaje, pues permite ligar la docencia con la actividad investigativa y con la actividad de extensión para relacionar al sujeto en formación con la realidad de su entorno y de sus semejantes, los hace sensibles a las necesidades sociales y les permite desarrollar el sentido del emprendimiento y la innovación en la solución a problemas de la sociedad.

\section{Conclusiones}

1. La pertinencia de los procesos que se desarrollan en el Laboratorio de Ciencias Naturales ha sido un elemento característico en las carreras que hacen uso de estos espacios y esta variable fue considerada como muy buena por estudiantes y docentes.

2. Los procesos y actividades de formación desarrolladas en el Laboratorio de Ciencias Naturales han aportado efectiva y eficientemente, favoreciendo el aprendizaje del estudiantado y al fortalecimiento de las capacidades de las y los docentes.

3. A pesar de una percepción positiva sobre la calidad en los procesos desarrollados, el estudiantado considera la necesidad de superar debilidades como la ampliación y habilitación de espacios y dotación de materiales, equipos y reactivos.

4. Los currículos y programas de asignaturas de las carreras que demandan el uso del Laboratorio de Ciencias Naturales no detallan el desarrollo y requerimientos para prácticas en estos.

5. La apertura del área técnico-académica "Laboratorio de Ciencias Naturales" ha influido de forma positiva en el cambio de actitud hacia la formación científico-técnica de la comunidad de aprendizaje, lo cual se traduce en mayores conocimientos, habilidades y capacidades.

\section{Agradecimiento}

Esta publicación obtuvo el financiamiento del Proyecto Ecosistemas Costeros, auspiciado por: Unión Europea, Dka Austria, Cooperación Austriaca para el Desarrollo y Horizontzooo.

\section{Lista de referencias}

Alonzo Blanqueto, C. G., \& Rodríguez Solís, G. (1990). El laboratorio en la enseñanza de las ciencias naturales: ¿Plato fuerte o plato de segunda mesa? Educación y Ciencia, 1 (1), 21-23. Recuperado de http://www.educacionyciencia.orgindex.phpeducacionycienciaarticleview8pdf 
Barberá, O., \& Valdés, P. (1996). El trabajo práctico en la enseñanza de las ciencias: Una revisión. Enseñanza de las Ciencias, 14(3), 365-379. Recuperado de http://www.raco.cat/index.php/ensenanza/article/ viewFile/21466/93439\%3Forigin\%3Dpublication_detail

Cardona, F. E. (2013). Las prácticas de laboratorio como estrategia didáctica. Universidad del Valle. Santiago de Cali, recuperado de http://bibliotecadigital.univalle.edu.co/bitstream/10893/6772/1/CD-0395428. pdf

Colado, J. (s.f.). El experimento docente dentro de la actividad de laboratorio. Su importancia en la educación científica de los estudiantes. Breves consideraciones sobre algunos modelos de aprendizaje que inciden en elexperimento. Instituto Superior Pedagógico "Enrique José Varona". La Habana, Cuba. Recuperado de http://fisica.cubaeduca.cu/index.php?option=com_content\&view=article\&id= 11429\%3Ael-experimento-docente-dentro-de-la-actividad-de-laboratorio\&catid=526\%3Afisica

Gobierno de Córdoba, Ministerio de Educación, Secretaría de Educación, Subsecretaría de Estado de Promoción de Igualdad y Calidad Educativa (2015). Materiales de laboratorio: recursos para enseñar y aprender Ciencias Naturales. Recuperado de http://psissuu.comsnow756docsmatlab_csn_eduprim

Flores, J., Caballero, M. \& Moreira, M. (2009). El laboratorio en la enseñanza de las ciencias: Una visión integral en este complejo ambiente de aprendizaje. Revista de investigación, 33 (68), 75-111. Recuperado de https://dialnet.unirioja.es/servlet/articulo?codigo=3221708

Hodson, D. (1994). Investigación y experiencias didácticas: Hacia un enfoque más crítico del trabajo de laboratorio. The Ontario Institute for Studies in Education, Toronto, Canadá. Enseñanza de las Ciencias, 12(3), 299-313. Recuperado de http://www.raco.cat/index.php/ensenanza/article/ viewFile/21370/93326

Hofstein, A. y Lunetta, V. N. (2004). The laboratory in science education: Science Education. 52, 201-217. Recuperado de http://gpquae.iqm.unicamp.br/gtexperimentacao.pdf

Kirschner, P.A. (1992). Epistemology, practical work y academic skills in science education. Science Education, 1, 273-299. Recuperado de http://link.springer.com/article/10.1007/BFo0430277

López, R. A. M. \& Tamayo, O. E. (2012). Las prácticas de laboratorio en la enseñanza de las ciencias naturales. Revista Latinoamericana de Estudios Educativos, 8 (1), 145-166. Recuperado de http://www. redalyc.org/pdf/1341/1341292560o8.pdf

Lugo, G. (2006). La importancia de los laboratorios. Construcción y tecnología, 15 (223), 20-22. Recuperado de http://www.imcyc.com/revistacto6/dico6/INGENIERIA.pdf

Pickering, M. (1993). The teaching laboratory through history. Journal of Chemical Education, 70(9), 699700. Recuperado de http://pubs.acs.org/doi/pdf/10.1021/edo70p699

Salcedo Torres, L. E., Villareal Hernández, M. E., Zapata Castañeda, P. N., Rivera Rodríguez, J. C., Colmenares Guluma, E., \& Moreno Romero, S. P. (2005). Las prácticas de laboratorio en la enseñanza de la química en educación superior. Proyecto de investigación, Universidad Pedagógica Nacional, Bogotá, Colombia. Recuperado de https://ddd.uab.cat/pub/edlc/edlc_a2005nEXTRA/edlc_a2005nEXTRAp2ogpralab.pdf

Urrea, G., Niño, J., García, J., Alvarado J., Barragán, G. \& Hazbón, O. (2013). Del aula a la realidad. La importancia de los laboratorios en la formación del ingeniero. Caso de estudio: ingeniería aeronáutica -Universidad Pontificia Bolivariana. Innovation in research and ingineering education: key factors for global competitiveness. World Ingineering Education Fórum (WEEF) llevado a cabo en Cartagena de Indias, Colombia. Recuperado de http://www.acofipapers.org/index.php/acofipapers/2013/paper/ viewFile/502/145 
URACCAN

URACCAN (2014). Plan estratégico institucional de URACCAN, 2015-2019: Resumen ejecutivo. Regiones Autónomas dela Costa Carie de Nicaragua.

URACCAN (2008). Proyecto Educativo Institucional. Costa Caribe de Nicaragua.

URACCAN (2004). Modelo Pedagógico de URACCAN. Costa Caribe de Nicaragua.

Weyrauch, V. (2012). Guía $N_{5}^{\circ}$ : Métodos de recolección de datos. En ¿Cómo monitorear y evaluar la incidencia en políticas públicas? Buenos Aires: CIPPEC. 